\title{
Investigation of Nosocomial Cluster Infections of Peripheral Venous Catheter-Related Bloodstream Infection
}

\author{
Mengying Qi ${ }^{1,2}$, Jinai $\mathrm{He}^{2,}$, Jiajie Yan $^{2}$ \\ ${ }^{1}$ School of Nursing, Jinan University, Guangzhou, China \\ ${ }^{2}$ The First Affiliated Hospital, Jinan University, Guangzhou, China
}

Email address:

qimengying0605@163.com (Mengying Qi),jiejyan@163.com (Jiajie Yan)

${ }^{*}$ Corresponding author

\section{To cite this article:}

Mengying Qi, Jinai He, Jiajie Yan. Investigation of Nosocomial Cluster Infections of Peripheral Venous Catheter-Related Bloodstream Infection. American Journal of Nursing Science. Vol. 9, No. 6, 2020, pp. 433-437. doi: 10.11648/j.ajns.20200906.18

Received: November 17, 2020; Accepted: November 27, 2020; Published: December 4, 2020

\begin{abstract}
Object: Determine the cause of nosocomial cluster infections of peripheral venous catheter-related bloodstream infection (PIVC-BSI), and provide advice on prevention, control and nursing measures. Method: A retrospective analysis of nosocomial cluster infections that occurred in a tertiary hospital on July 28-30, 2019. Blood samples from patients suspected of PIVC-BSI were retained for bacterial culture. The clinical data of the patients, environmental hygiene indicators were collected. Results: All 8 patients had chills within 0.5 - 3.5 hours after intravenous infusion, and the highest body temperature reached $41^{\circ} \mathrm{C}$. The peripheral blood samples of 4 patients were cultured as Burkholderia cepacia infection, and the eluate from the catheter tip of 2 patients cultured the same bacteria as the blood culture. The humidity in the treatment preparation room was $60 \%-80 \%$. There was mold in the sterile storage cabinet. Conclusion: The nosocomial cluster infection of PIVC-BSI was caused by Burkholderia cepacia colonizing the tip of the peripheral intravenous catheter, and the high air humidity was the most likely factor. The ability of nurses to identify and respond to PIVC-BSI as well as the infection control management level of each department still needs to be improved.
\end{abstract}

Keywords: Peripheral Intravenous Catheter, Peripheral Intravenous Catheter-Related Bloodstream Infection, Burkholderia Cepacian, INFECTION Control

\section{Introduction}

Infection events are common adverse events in hospitals that seriously threaten the lives and health of patients, which often occur in departments with more intrusive operations, such as intensive care units (ICUs). Previous studies have paid more attention to central venous catheter-related bloodstream infections. Compared with other venous catheter-related bloodstream infections, peripheral venous catheter-related bloodstream infections (PIVC-BSI) have a lower risk. However, the peripheral intravenous catheter (PIVC) puncture operation is the most common invasive procedure in clinical practice. More than one billion patients are implanted with PIVC every year worldwide [1]. Due to the large number of PIVCs used, the absolute infection rate of PIVCs is close to the absolute infection rate of other catheters [2]. Once a bloodstream infection occurs, it will increase the patients' discomfort, mortality, hospitalization time, and cost [3, 4]. Therefore, PIVC-BSI has received more attention in recent years. In 2006, a meta-analysis of 200 studies from 1966 to 2005 by Maki DG et al. found that the incidence of PIVC-BSI was 0.5 per 1000 PIVC days in ICUs in Australia, Italy, and the United States [2]. A six-year multicenter prospective surveillance study of PIVC-BSI from September 1, 2013 to May 31, 2019 conducted by members of the International Nosocomial Infection Control Consortium (INICC) in 727 intensive care units in 42 countries identified 1,789 PIVC-BSIs for an overall rate of 2.41 per 1,000 PIVC days. The mortality of patients with PIVC-BSI was $12.21 \%$, and the average length of stay was 7.11 days [5]. It is difficult to identify the infection in the early stage timely because nosocomial cluster infection of peripheral venous catheter-related bloodstream infection is less likely to occur. Microorganisms can come into contact with PIVC in various ways, such as through contaminated infusion sets or catheters, the skin near the entrance of the 
catheter, or the staff's own flora [6]. Afterwards, microorganisms usually colonize the entrance of the catheter and the junction with the skin. During intravenous infusion, the microorganisms may be flushed into the blood and cause bloodstream infection [7]. If the route of infection is not detected and cut off in time, it will lead to an outbreak of PIVC-BSI and may even bring more serious consequences. This article aims to analyze the causes of nosocomial cluster infections of PIVC-BSI.

\section{Method}

A retrospective analysis of nosocomial cluster infections that occurred in a tertiary hospital on July 28-30, 2019. Catheter-related bloodstream infection refers to patients with symptoms of bacteremia or mycological infection during indwelling of intravascular catheters or within 48 hours after removement, accompanied by clinical symptoms such as shivers and fever $\left(>38^{\circ} \mathrm{C}\right)$, and excludes infections caused by other infection sources [8]. Blood samples from patients suspected of PIVC-BSI were retained, the clinical data of the patients, including gender, age, underlying diseases, diagnosis, name of surgery, operation time, laboratory test results, hospital stay and prognosis were collected. Environmental hygiene indicators, including the results of bacterial culture of the surface of objects, the hands of medical staff, and the air in the operating room, ward, and treatment preparation room were collected.

\section{Results}

\subsection{Characteristics of Participants}

On July 28-30, 2019, 8 patients in the orthopedics ward of a tertiary hospital had shivers with no obvious cause in the midday. The time of shiver of the 8 patients was 3-12 days after operation, and the highest body temperature was up to $38.4-41^{\circ} \mathrm{C}$. Median patient age was 58.5 (31.0-72.0) years. Of the 8 patients, 6 were female and 2 were male. In addition, there was one patient with hypoproteinemia and one patient with type 2 diabetes. All 8 patients had undergone orthopedic surgery, including: 3 cases of artificial knee replacement, 3 cases of artificial hip replacement, 1 case of artificial femoral head replacement, and 1 case of hand trauma (Table 1). There were no obvious signs of infection such as redness, swelling, or exudation in the wounds at the surgical site. The drugs used in the operations are mainly anesthetics, and the drugs used by the patients after surgery were all mixed by the pharmacy intravenous admixture services (PIVAS). No obvious abnormalities were found in the patients' chest X-ray and electrocardiogram (ECG) examination. Most of the patients' inflammatory-related indicators increased to varying degrees and exceeded the normal range. All 8 patients had signs and symptoms of bloodstream infection after flushing and locking the PIVCs, and all patients had no other indwelling catheters except PIVCs.

The PIVCs of the patients were removed after symptoms of bloodstream infection, and the tip of the catheters was cut $1-2$ centimetres, while retaining used and unused infusion devices and drugs by the patients for bacterial culture. The peripheral blood samples of 7 patients were retained for aerobic and anaerobic bacterial culture. Four of the patients' peripheral blood samples cultured Burkholderia cepacian, and the median time to positive culture of peripheral blood was 25.7 (23.0-39.7) h. Two cases of catheter tip eluate cultured the same bacteria as the blood samples, suggesting an outbreak of nosocomial infection (Table 2). The results of the drug susceptibility test showed sensitivity to ceftazidime.

\subsection{Outcomes}

All 8 patients improved after treatment and have been discharged from the hospital. The average length of hospital stay was extended by 9.25 days, and the average cost increased by RMB 6,548.55. The emergency plan was activated immediately after the incident, and various prevention and control measures were actively implemented, which effectively controlled the infection outbreak of infection. No unexplainable patient fever has occurred so far.

\subsection{Environmental Hygiene Monitoring}

The infection control team collected samples from the ward, treatment preparation room, and operating room, mainly including air, skin disinfectant, surface of objects, rags, the inner surface of the sink, the surface of the hands of medical staff, surgical instruments and the same batch of unopened samples of intravenous solution and PIVCs. The staff of the department immediately cleaned and disinfected all areas on the first day of the incident. Since the cleansing and disinfection had been carried out before the sampling of the infection control team, burkholderia cepacian was not detected in the culture results. However, during the investigation process, it was found that the temperature of the treatment preparation room fluctuated between $20^{\circ} \mathrm{C}$ and $22^{\circ} \mathrm{C}$, and the air humidity fluctuated between $60 \%$ and $80 \%$ throughout the summer. The surface of the sterile articles was wet, and the storage cabinet for the articles had mold growth.

Table 1. Characteristics of participants.

\begin{tabular}{l|l}
\hline Characteristic & All cases $(\mathbf{N}=\mathbf{8})$ \\
\hline $\begin{array}{l}\text { Age, years } \\
\text { Median }\end{array}$ & 58.5 \\
$\begin{array}{l}\text { Range } \\
\text { Sex, } \mathrm{n} \%\end{array}$ & $31.0-72.0$ \\
$\begin{array}{l}\text { Male } \\
\text { Underlying condition, } \mathrm{n} \%\end{array}$ & $2(25.0)$ \\
$\begin{array}{l}\text { Hypoproteinemia } \\
\text { Type } 2 \text { diabetes }\end{array}$ & $1(12.5)$ \\
$\begin{array}{l}\text { Operation, } \mathrm{n} \% \\
\text { Artificial knee replacement }\end{array}$ & $1(12.5)$ \\
$\begin{array}{l}\text { Artificial hip replacement } \\
\text { Artificial femoral head replacement }\end{array}$ & $3(37.5)$ \\
Hand trauma & $3(37.5)$ \\
Time to positive culture of peripheral blood, $\mathrm{h}$ & $1(12.5)$ \\
\hline $\begin{array}{l}\text { Median } \\
\text { Range }\end{array}$ & $1(12.5)$ \\
\hline
\end{tabular}


Table 2. Bacterial culture results of participants' peripheral blood and catheter tip eluate.

\begin{tabular}{llllll}
\hline Date & Cases No. & End of infusion & Shivers/fever & Bacterial culture of peripheral blood & Bacterial culture of catheter tip eluent \\
\hline July 28th & 1 & $09: 30$ & $13: 00$ & $\mathrm{~B}$ & No \\
& 2 & $10: 00$ & $13: 06$ & No & No \\
& 3 & $10: 50$ & $13: 07$ & $\mathrm{~N}$ & No \\
July 29th & 4 & $10: 40$ & $11: 40$ & $\mathrm{~N}$ & $\mathrm{~N}$ \\
& 5 & $11: 00$ & $11: 30$ & $\mathrm{~B}$ & $\mathrm{~B}$ \\
& 6 & $11: 00$ & $12: 00$ & $\mathrm{~N}$ & $\mathrm{~N}$ \\
July 30th & $\mathbf{6}$ & $11: 30$ & $14: 00$ & $\mathrm{~B}$ & $\mathrm{~N}$ \\
\hline
\end{tabular}

Note. B, burkholderia cepacian; $\mathrm{N}$, negative; No, not sent for inspection.

\section{Discussion}

\subsection{Preliminary Judgment on PIVC-BSI}

There are few reports and studies on PIVC-BSI in China due to the low incidence. In the nosocomial cluster infections, it was first considered whether the source of infections was due to insufficient air cleanliness, equipment contamination, or improper operation. The burden of complications associated with peripheral intravenous use is underestimated, due to the broad use, inconsistent coding, and lack of mandatory reporting of PIVCs [4], we failed to consider the cluster infections caused by PIVCs in time. In these cases, the patients all experienced shivers during the indwelling of PIVCs, body temperatures were greater than $38^{\circ} \mathrm{C}$, which was consistent with the clinical symptoms of catheter-related bloodstream infection. In addition, medical staff gradually ruled out the possibility of other infection routes. (1) Surgical fever refers to the reaction that occurs after the absorption of tissue decomposition products, local exudation, and bleeding due to the destruction of surgical operation. Postoperative patient's body temperature can be slightly increased, the fluctuation range is $0.5-1^{\circ} \mathrm{C}$, generally does not exceed $38.5^{\circ} \mathrm{C}$. Eight patients had shivers 3-12 days after surgery, and their body temperature exceeded $38.5^{\circ} \mathrm{C}$. Surgical fever patients usually did not have shivers, so we excluded the factors of surgical fever. (2) Since all 8 patients have completed orthopedic surgery, and 7 of them have implanted artificial joints, it is suspected to be caused by implants or contamination of the operating room. However, there were no obvious signs of infection such as redness and exudation in the wound at the surgical site, and the implants of 7 patients were from different batches. The investigation found that other patients who used the same batch of products or used the same operation room had no signs of bloodstream infection, so the factors of implant or operation room contamination were excluded. On the second day of the infection, investigators noticed that all patients developed symptoms of infection shortly after the end of the intravenous infusion. The time from exposure to infection of patients with bloodstream infection is usually very short (0.5-2 days) [9], and all patients had no indwelling catheter except PIVC, so it was suspected to be PIVC-BSI. After retaining the PIVCs of some patients and culturing the eluate of PIVCs, it was determined to be PIVC-BSI.

\subsection{Find out the Cause}

The drugs used by the patients after surgery were all mixed by the PIVAS. After screening more than 1,700 patients in other wards of the hospital, it was found that there was no patient's cluster of shivers and fever. The causes of the patients' fever could be reasonably explained in other wards, so it was considered causing by the internal reasons of the ward. Burkholderia cepacia is usually isolated from natural soil, water, plants, and animals. It is an aerobic, non-fermentative Gram-negative bacillus often found in contaminated water, pipes, and instruments. It is also one of the common sources of nosocomial infections $[9,10]$. Throughout the summer, the air humidity in the treatment preparation room fluctuates between $60 \%$ and $80 \%$, which is suitable for the growth and reproduction of Burkholderia cepacian. When the high temperature lasts for a long time and the air conditioning temperature in the treatment preparation room is set too low, once the door of the treatment preparation room is opened, the cold and hot air inside and outside the room will produce condensate, which will increase the air humidity and create conditions for bacterial reproduction, it will also affect the dry storage of sterile items. The investigation found that the surface of the sterile items in the treatment preparation room was wet, and the storage cabinet storing the infusion set had mold growth. Since the items have been replaced and cleaned before sampling, no real source of infection was found in this cultivation. Nevertheless, the investigation speculates that the infusion tool is more likely to be contaminated due to moisture. In addition, the implementation of infection management in the department is not strong enough, and the implementation of various infection prevention and control measures in the department should be supervised and inspected regularly. After the disinfection of the environment, the adjustment of humidity, and the complete replacement of the infusion tool, no patients developed cluster infections.

\subsection{Prevention and Control Measures}

Environmental disposal. (1) Strengthen environmental cleaning and disinfection every day. Wet cleaning should be used, which mainly includes the floor, bed unit, and surface of objects. The floor could be cleaned in the shape of "N", and the surface of the article could be wiped in the shape of "S". Follow the unitized operation when cleaning and disinfecting 
the bed unit, which means that one cleaning cloth matches with one bed unit and cannot be used interchangeably. For high-frequency contact surfaces, such as consoles, storage cabinets, bed rails, door handles, etc., cleaning and disinfection were more frequently than low-frequency contact surfaces. (2) Regulate the temperature and humidity in the treatment preparation room. Record the temperature and humidity of the treatment preparation room in each shift, set the air conditioner temperature to $26^{\circ} \mathrm{C}$, and when the air humidity exceeds $60 \%$, open the door for ventilation or use a dehumidifier to reduce the air humidity. (3) Replace the contaminated items. When it is suspected that the PIVC-BSI incidents may be directly transmitted through wet and contaminated PIVCs, all infusion-related items in the treatment preparation room should be replaced, including infusion sets, infusion needles, and infusion patches.

Management improvement. (1) Strengthen the monitoring of the environment and patients. The nurses should monitor patients in the ward every day for signs of infection, monitor the temperature and humidity of the treatment preparation room, and whether the storage of sterile items is intact. The head nurses check the implementation of monitoring, cleaning, and disinfection weekly. (2) The formulation and implementation of standardized operating procedures. The hospital formulates disinfection guidelines for treatment preparation rooms, treatment rooms, and disposal rooms, and formulates standard operating procedures for the use of dirt elevators, and implements and manages them in accordance with unified standardized operating procedures. (3) Update of nursing emergency management plan. To strengthen the attention of all clinical nurses to PIVC-BSI, the intravenous treatment specialist team need to develop a standardized emergency procedure for fever response to infusion, especially when catheter-related bloodstream infection is suspected.

Strengthen training. The medical staff of the whole hospital, especially the clinical nurses should be taught about the "Management Regulations for Environmental Surface Cleaning and Disinfection of Medical Institutions" promulgated by the National Health Commission of the People's Republic of China in 2016. Strengthen the training of nurses on PIVC-BSI identification and emergency response, and instruct logistics personnel on how to effectively clean and disinfect the ground and surface of objects and deal with dirt.

Since it is difficult to prevent microorganisms from colonizing the skin near the entrance of the catheter, nurses can only reduce the reproduction of microorganisms through various measures. The principle of aseptic operation must be followed during the puncture to prevent microorganisms from entering the blood through the hands of medical personnel and contaminated infusion devices. We recommend that skin antisepsis could be performed using $2 \%$ chlorhexidine in $70 \%$ isopropyl alcohol before puncture [11]. In addition, it is recommended to use needle-free connectors to reduce the chance of blood exposure [12]. It is recommended to disinfect the skin and change the dressing in time when the dressing of the fixed catheter is loose or contaminated by blood or sweat.

\section{Conclusion}

Although PIVC-BSI is mainly caused by Staphylococcus aureus, in recent years, there have been some reports of nosocomial infections caused by Burkholderia onion in China, most of which have not found a clear source of infection [10, 13, 14]. For uncommon strain infections, we should find out the characteristics of the strains in a timely manner, delete the cause of the infection in a targeted manner, and formulate prevention and control measures and treatment plans. Once bacteria colonize the catheter tip, the long-term application of antibiotics may increase the drug resistance of the strains and may also lead to new colonization [15]. Every clinical nurse beside nurses specializing in intravenous therapy should have the ability to identify PIVC-BSI and implement effective measures in strict accordance with the standardized emergency procedure for fever response to infusion, and correctly collect relevant samples as soon as possible. Although the source of infection was not clear, the potential factors were found through the examination of the department environment and the investigation of personnel. By adopting a series of countermeasures, we prevented the more serious consequences that PIVC-BSI might bring in a timely manner and summed up the experience for future department infection management.

\section{Declaration of Conflicting Interests}

The authors declare that they have no competing interests.

\section{Funding}

This work was unfunded.

\section{References}

[1] Alexandrou, E., Ray - Barruel, G., Carr, P. J., Frost, S., Inwood, S., Higgins, N.,... \& Rickard, C. M. (2015). International prevalence of the use of peripheral intravenous catheters. Journal of hospital medicine, 10 (8), 530-533.

[2] Maki, D. G., Kluger, D. M., \& Crnich, C. J. (2006, September). The risk of bloodstream infection in adults with different intravascular devices: a systematic review of 200 published prospective studies. In Mayo Clinic Proceedings (Vol. 81, No. 9, pp. 1159-1171). Elsevier.

[3] Barnett, A. G., Page, K., Campbell, M., Martin, E., Rashleigh-Rolls, R., Halton, K.,... \& Graves, N. (2013). The increased risks of death and extra lengths of hospital and ICU stay from hospital-acquired bloodstream infections: a casecontrol study. BMJ open, 3 (10).

[4] Lim, S., Gangoli, G., Adams, E., Hyde, R., Broder, M. S., Chang, E.,... \& Danker III, W. (2019). Increased Clinical and Economic Burden Associated With Peripheral Intravenous Catheter-Related Complications: Analysis of a US Hospital Discharge Database. INQUIRY: The Journal of Health Care Organization, Provision, and Financing, 56, 0046958019875562. 
[5] Rosenthal, V. D., Belkebir, S., Zand, F., Afeef, M., Tanzi, V. L., Al-Abdely, H. M.,... \& Elahi, N. (2020). Six-year multicenter study on short-term peripheral venous catheters-related bloodstream infection rates in 246 intensive units of 83 hospitals in 52 cities of 14 countries of Middle East: Bahrain, Egypt, Iran, Jordan, Kingdom of Saudi Arabia, Kuwait, Lebanon, Morocco, Pakistan, Palestine, Sudan, Tunisia, Turkey, and United Arab Emirates-International Nosocomial Infection Control Consortium (INICC) findings. Journal of Infection and Public Health.

[6] Chernecky, C., \& Macklin, D. (2014). The role of IV needleless connectors and IV complication management and prevention. Advances in Research, 195-206.

[7] Choudhury, M. A., Sidjabat, H. E., Zowawi, H. M., Larsen, E., Paterson, D. L., McMillan, D. J., \& Rickard, C. M. (2019). Skin colonization at peripheral intravenous catheter insertion sites increases the risk of catheter colonization and infection. American Journal of Infection Control, 47 (12), 1484-1488.

[8] Montecalvo, M. A., McKenna, D., Yarrish, R., Mack, L., Maguire, G., Haas, J.,... \& Watson, A. (2012). Chlorhexidine bathing to reduce central venous catheter-associated bloodstream infection: impact and sustainability. The American journal of medicine, 125 (5), 505-511.

[9] Zou, Q., Li, N., Liu, J., Li, X., Wang, Z., Ai, X.,... \& Hu, Y. (2020). Investigation of an outbreak of Burkholderia cepacia infection caused by drug contamination in a tertiary hospital in China. American Journal of Infection Control, 48 (2), 199-203.

[10] Peng, F., Zhong, L. L., Lin, X. J., Chen, M., \& Zhou, M. (2018).
Burkholderia cepacia infection in children: a clinical analysis of 16 cases. Zhongguo Dang dai er ke za zhi= Chinese Journal of Contemporary Pediatrics, 20 (2), 112-115.

[11] De Plato, F., Fontana, C., Gherardi, G., Privitera, G. P., Puro, V., Rigoli, R.,... \& Viale, P. (2019). Collection, transport and storage procedures for blood culture specimens in adult patients: recommendations from a board of Italian experts. Clinical Chemistry and Laboratory Medicine (CCLM), 57 (11), 1680-1689.

[12] Sengul, T., Guven, B., Ocakci, A. F., \& Kaya, N. (2020). Connectors as a risk factor for blood-associated infections (3-way stopcock and needleless connector): A randomized-experimental study. American Journal of Infection Control, 48 (3), 275-280.

[13] Mermel, L. A. (2017). Short-term peripheral venous catheterrelated bloodstream infections: a systematic review. Clinical Infectious Diseases, 65 (10), 1757-1762.

[14] Chien, Y. C., Liao, C. H., Sheng, W. H., Chien, J. Y., Huang, Y. T., Yu, C. J., \& Hsueh, P. R. (2018). Clinical characteristics of bacteraemia caused by Burkholderia cepacia complex species and antimicrobial susceptibility of the isolates in a medical centre in Taiwan. International Journal of Antimicrobial Agents, $51(3), 357-364$.

[15] Duan, J., Kang, J., Han, T., Ma, Y., Guo, Q., Song, Y.,... \& Zhang, R. (2017). Prevalence of hospital acquired Burkholderia cepacia infection and its antimicrobial susceptibility in a Chinese hospital. Pakistan Journal of Pharmaceutical Sciences, 30 (2). 